\title{
LA VISITA D'IMPRESA. DA ATTRAZIONE A PRODOTTO TURISTICO
}

Luca Savoja,

University of Turin, Italy

Resumen: Visitar empresas. La transformación de una atracción en producto turístico

El Turismo Industrial es un concepto complejo que abarca muchas actividades turísticas, una de ellas es el llamado Turismo de Industria Viva que toma sustancia en el visitar por placer compañías y empresas industriales mientras están funcionando. El Turismo de Industria Viva es una actividad encaminada a proporcionar un conocimiento directo de los procesos y productos típicos y representativos del territorio local.

Puede haber varios tipos de motivaciones para esta tipología turística, desde las más orientadas al turismo escolar y/o de estudio hasta las formas más lúdicas en las el deseo de conocer se acopla con la posibilidad de probar, experimentar y comprar productos.

Este artículo se centra en una discusión sobre las principales características del Living Industry Tourism, de su potencial y sus debilidades como producto comercial en el área del turismo.

Además, para mostrar cómo es posible transformar esta atracción potencial en una oportunidad concreta para los turistas, en el artículo se presentan algunos detalles de un caso innovador en el que las visitas de empresa se van ofreciendo al segmento de los turistas individuales de la misma manera de las otras atracciones turísticas de una gran ciudad como es el caso de Turín en Italia.

Palabras clave:Turismo de Industria Viva, Visitas de Empresa, Prácticas Turísticas, Percepciones

Resumo: Visitar empresas. A transformación dunha atracción en produto turístico

$\mathrm{O}$ "turismo industrial" é un concepto complexo que abarca moitas actividades turísticas. Unha delas é o chamado "turismo de industria viva", que toma substancia no visitar por pracer compañías e empresas industriais mentres están funcionando. O turismo de industria viva é unha actividade encamiñada a proporcionar un coñecemento directo dos procesos e produtos típicos e representativos do territorio local.

Pode haber varios tipos de motivacións para esta tipoloxía turística, desde as máis orientadas ao turismo escolar e/ou de estudo ata as máis lúdicas, en que o desexo de coñecer se combina coa posibilidade de probar, experimentar e comprar produtos.

Este artigo céntrase nunha discusión sobre as principais características do Living Industry Tourism, do seu potencial e as súas debilidades como produto comercial na área do turismo. 
Ademais, para mostrar como é posible transformar esta atracción potencial nunha oportunidade concreta para os turistas, no artigo preséntanse algúns detalles dun caso innovador en que as visitas de empresa se van ofrecendo ao segmento dos turistas individuais do mesmo xeito que as outras atraccións turísticas dunha gran cidade como Turín (Italia).

Palabras clave: Turismo de industria viva, visitas de empresa, prácticas turísticas, percepcións

\begin{abstract}
Company Guided Tours. Transforming an attraction in touristic product
"Industrial Tourism" is complex concept encompassing many different tourist activities, one of them is the named Living Industry Tourism that takes the forms on touring companies while they are operating. Living Industry Tourism is an activity aimed at providing direct knowledge of processes and products typical to or representative of the local territory. There can be various kinds of motivations for this type of tour: strictly for learning or the more 'fun' sort in which learning about a company comes with the chance to try or to buy the products.

This paper is focused on a discussion on the main features of Living Industry Tourism and its potential and weaknesses as product marketable in the touristic arena.

To show how is possible to transform the Living Industry Tourism in a concrete opportunity for toursits, the paper present even some details of an innovative case in which Company Guided Tours are offered to the segment of individual tourists in the same offer as the other tourism attractions in a major tourism destination like Turin in Italy.
\end{abstract}

Keywords: Living Industry Tourism, Company Guided Tours, Touristic Patterns, Perceptions

Il significativo aumento di importanza del turismo è un fenomeno contemporaneo diffuso e ben conosciuto.

Tale tendenza non è solo determinata dall'aumento dei flussi che risulta costante negli anni e sembra, se si considera l'insieme dei movimenti turistici mondiali dell'ultimo biennio, quasi indipendente dall'attuale crisi globale; ciò che emerge con altrettanta chiarezza è anche l'allargamento spaziale degli interessi degli operatori turistici e dei loro clienti.

In effetti oggi si può affermare che ogni chilometro quadrato della Terra (e non solo, visto che sono sempre più numerose le possibilità offerte dal cosiddetto "turismo spaziale" ) possiede una qualche valenza turistica.

Tuttavia, mentre il processo di "colonizzazione turistica" del Pianeta può dirsi quasi concluso, non altrettanto si può dire per la messa a valore dei sight che compongono il patrimonio turistico di un luogo.

Le attrazioni potenziali, vale a dire gli oggetti, gli spazi, i beni materiali e quelli immateriali sono, di norma, molto più numerose di quelle che vengono offerte per il consumo dei visitatori. La messa in scena turistica è sovente limitata ad aspetti "tradizionali" dell'heritage (come musei, monumenti, skyline, architetture urbane) e ad alcuni elementi della back region (come il folclore, i prodotti tipici, l'arte popolare, le produzioni artigianali, il reticolo del commercio tradizionale) supportati da un insieme di beni/servizio pensati ed offerti per il tempo libero (Jansen Verbeke, 1996).

1 Il volo spaziale è oggi ritenuto un settore commerciale interessante da parte di diversi "tour operator". E' del Novembre 2011 la notizia che la Virgin Galactic del gruppo Virgin di Richard Branson prevede di mettere in servizio una astronave "turistica" nel 2012. Il piano dell'offerta prevede che i viaggiatori si sottopongano ad un addestramento di soli tre giorni prima del lancio, che potrebbe portarli ad oltre 130.000 metri di quota. 
In tempi più recenti il processo di tourismification ha interessato anche quei manufatti ed edifici industriali che, dopo aver esaurito la loro funzione "produttiva", rappresentano strutture importanti e "ben visibili" nel panorama dei luoghi. Basti pensare alle zone ex industriali che caratterizzano quasi tutte le città europee e che, di norma, si trovano in zone ormai non più periferiche delle stesse.

In questo senso l'affermazione del turismo industriale, inteso come attività che trova la sua ragion d'essere nel visitare e conoscere testimonianze industriali del passato è una tipologia già consolidata. Dall'archeologia industriale, ai musei d'impresa ad esperienze dirette di recupero di tradizioni manifatturiere peculiari, il turismo industriale è, oggi, una nicchia ben caratterizzata nel panorama delle pratiche turistiche più diffuse.

Allo stesso modo, le tante esperienze di riconversione e riqualificazione urbana di molte aree industriali dismesse trasformate in luoghi per il tempo libero (musei, parchi a tema, aree per manifestazioni, complessi fieristici, ....), hanno collocato l'industria (o meglio le infrastrutture ex industriali) all'interno della scenografia turistica soprattutto per quanto riguarda il turismo urbano.

Molti edifici industriali, un esempio su tutti è la fabbrica automobilistica del Lingotto a Torino, da contenitori della produzione e dell'ingegno industriale, sono oggi luoghi privilegiati per il leisure time e per la pratica di varie attività turistiche.

Per quanto riguarda invece l'industria "viva", vale a dire siti produttivi funzionanti che vengono visitati con finalità conoscitive, informative e ludiche (o combinazioni di queste motivazioni), il processo di messa in scena turistica può dirsi, almeno in Europa, ancora in corso (Morice, 2006).

E' ben vero che la visita ad alcune categorie di "industrie vive" sono già da tempo ben presenti nel paniere standard del turista contemporaneo tanto che la loro fruizione configura specifiche forme turistiche. E' il caso delle cantine e di alcune industrie alimentari ed aziende agricole che alimentano il grande settore del turismo enogastronomico o quello di alcune aziende di abbigliamento o di altre tipologie di beni di consumo (cosmetici, oggettistica, accessori solo per citare alcuni esempi) che sono l'elemento fondamentale per un'altra modalità turistica, in forte crescita, rappresentata dallo shopping tourism.

Tuttavia il turismo d'impresa oggi è in espansione per via dell'aumento di attenzione verso le industrie in quanto "attrazioni", vale a dire intese come luoghi di interesse turistico al di là degli obiettivi consumistici riguardanti i loro prodotti. In questo senso il turismo d'impresa può dirsi caratterizzato da un interesse per il processo produttivo.

I motivi per questa espansione anche verso luoghi "non turistici" come le industrie in attività sono diversi e legati alle specificità dei vari segmenti di turisti (turismo scolastico, turismo formativo/professionale, alcune parti del turismo congressuale), tuttavia se si considera il turismo "generico" è possibile individuare due macrotendenze che stanno favorendo l'entrata dell'industria nell'offerta di numerosi territori. 
La prima è determinata dalle modificazioni in senso postfordista delle scelte turistiche, che tendono a sostituire l'unica vacanza annuale concentrata in località marine con più periodi di vacanza differenziati e trascorsi in varie località. Tale tendenza ha determinato la crescita della mobilità durante il tempo libero e favorito la maggiore attenzione turistica verso altri luoghi e la loro collocazione nel paniere dei consumi turistici più diffusi (Farrell \& Twining-Ward, 2004).

La possibilità di configurare un sito produttivo funzionante come attrazione turistica è data dalla capacità di offrirlo in modo variegato ed articolato alle diverse popolazioni turistiche che la visitano. Se, per altre attrazioni la messa in scena turistica determina una inevitabile falsificazione delle stesse, per i luoghi del turismo d'impresa tale rischio sembra scongiurato (proprio per la natura stessa delle imprese che non possono essere concepite per la fruizione turistica) così, le industrie sono, oggi, le attrazioni che meglio soddisfano uno degli obiettivi prioritari del turista moderno che è la ricerca di qualcosa di "autentico". All'interno di un offerta turistica sempre più ampia ma, per certi versi, sempre più subdolamente standardizzata, essa può rappresentare una "esperienza" originale e, proprio per questo, di pregio.

E' stato rilevato che numerose città trovano nello sviluppo del turismo industriale uno strumento primario per le proprie strategie di sviluppo locale; specialmente quelle città, che dopo aver perso il loro carattere monoindustriale, stanno ricostruendo la loro identità anche grazie all'incremento dell'offerta turistica (Van den Berg, 2010).

La seconda macrotendenza è dovuta alle trasformazioni che la postmodernità ha imposto al turismo (o più in generale al tempo libero degli individui delle società occidentali). Anthony Giddens (2000) ha notato che la modernità consiste essenzialmente in "meccanismi di sradicamento" che dilatano le relazioni sociali sia nel tempo sia nello spazio, frantumando così i confini normativi da tempo riferimento per le persone.

Tuttavia, al di là dell'ampio dibattito sull'essenza delle società contemporanee, in generale vi è accordo sul fatto che le trasformazioni più significative dell'agire sociale sono date dal superamento di molti confini fisici e simbolici che fornivano agli individui il contesto entro il quale costruire il significato alle loro azioni. Questo processo di superamento dei confini di significato è ormai pervasivo e trova un'area di elezione nell'ambito dei comportamenti di consumo all'interno dei quali è possibile ricondurre quelli turistici.

Una quota crescente di individui si sente attratta da proposte turistiche fondate sul prolungamento della quotidianità intesa come "sperimentazione" affiancata all'idea di "novità". In questo senso dunque, da turista il visitare le imprese pare assai congruente con tale tendenza in quanto facilita le relazioni fondate sul fare e sul conoscere (Zarate Martin, 2010).

Così fare turismo "dentro" le imprese (cioè un luogo elettivo di negazione della dimensione del divertimento e della loisir) non è più incoerente e non assume più dei connotati turisticamente negativi; al contrario può soddisfare, più di quanto possono farlo altre attività turistiche, la ricerca della differenziazione che è, vale ricordarlo, l'essenza ultima del consumo contemporaneo. 


\section{TURISMO INDUSTRIALE E LIVING INDUSTRY TOURISM}

Come detto, sotto l'etichetta di "Turismo Industriale" si possono ricondurre numerose attività; la stessa definizione del WTO conferma la complessità del concetto ${ }^{2}$.

All'interno di questa tipologia turistica si possono individuare tre principali modelli, tra loro complementari e, a loro volta, collegati ad altre tipologie turistiche in maniera articolata. La tabella successiva sintetizza le relazioni esistenti tra le varie tipologie turistiche.

\begin{tabular}{|c|c|c|c|c|c|c|}
\hline $\begin{array}{l}\text { INDUSTRIAL } \\
\text { HERITAGE } \\
\text { TOURISM }\end{array}$ & 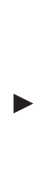 & $\begin{array}{c}\text { SPAZI } \\
\text { INDUSTRIALI } \\
\text { RICONVERTITI } \\
\text { PER IL TEMPO } \\
\text { LIBERO }\end{array}$ & $\triangleright$ & $\begin{array}{l}\text { LIVING } \\
\text { INDUSTRY } \\
\text { TOURISM }\end{array}$ & $=$ & $\begin{array}{c}\text { TURISMO } \\
\text { INDUSTRIALE }\end{array}$ \\
\hline$\nabla$ & & $\nabla$ & & $\nabla$ & & \\
\hline \multirow[t]{3}{*}{$\begin{array}{c}\text { Turismo } \\
\text { Culturale }\end{array}$} & & $\begin{array}{l}\text { Leisure } \\
\text { tourism }\end{array}$ & & $\begin{array}{c}\text { Turismo } \\
\text { enogastronomico }\end{array}$ & & \\
\hline & & & & $\&$ & & \\
\hline & & & & $\begin{array}{l}\text { Shopping } \\
\text { Tourism }\end{array}$ & & \\
\hline
\end{tabular}

Aree di complementarietà; $\boldsymbol{\nabla}$ Aree di parziale sovrapposizione

Il primo modello è quello del turismo del patrimonio industriale e dell'archeologia industriale (Industrial Heritage Tourism) che si caratterizza per l'interesse verso manufatti e simboli del passato manifatturiero. Industrie o, talvolta interi siti industriali vengono "messi in scena" per favorirne la fruizione da parte di visitatori di vario genere, anche all'interno di strutture come i musei d'impresa. Questa forma turistica è riconducibile all'area del turismo culturale.

Il secondo è quello degli spazi industriali riconvertiti per le pratiche del tempo libero; luoghi industriali abbandonati vengono ripensati e riproposti come

2 "Industrial heritage for tourism as tourism resources and assets composed primarily by industrial establishments, past and present, and their creations, as well as industry-related buildings, architectural ensambles and landscapes, equipment, machines and tools, other item used in production and handicrafts, skills and technologies, as well as industry-related lifestyles, customs traditions, events, creative arts and objects of art inspired by industrial and technical development and testifying to such development, both past and present", Dichiarazione di Astana, UNWTO, 2009. 
"contenitori" per il leisure. In questo la "ex-fabbrica" diviene un contenitore turistico e la sua visita è accessoria alla fruizione di attrazioni specifiche (musei, esposizioni, eventi, ...) ed all'uso del tempo libero.

Il terzo modello turistico è quello che pone al centro dell'interesse la visita ad imprese in attività e può essere definito come "Living Industry Tourism"3.

Il Living Industry Tourism si sostanzia come un'attività orientata alla conoscenza diretta di processi e prodotti "tipici" e rappresentativi del territorio di riferimento. La motivazione alla visita può essere di varia natura: da quella prettamente conoscitiva (Turismo scientifico, Educational Tourism) a quella più ludica che affianca alla conoscenza dell'impresa la possibilità di provare ed acquistare i prodotti (in questo modo, come discusso in precedenza, il Living Industry Tourism si sovrappone parzialmente allo Shopping Tourism ed al turismo enogastronomico).

Questo paper si occupa di quest'ultimo modello e, a partire da un discussione sulle sue principali caratteristiche, presenta un case history dove le visite d'impresa vengono offerte ai turisti individuali allo stesso modo e attraverso gli stessi canali di altre attrazioni che compongono l'offerta di una destinazione.

Questa proposta, che sta riscuotendo un grande successo di pubblico, trasforma un'opportunità normalmente riservata a gruppi organizzati e variamente motivati, in un prodotto turistico tout court. Il turismo d'impresa entra così nei meccanismi diffusi di fruizione turistica, andando oltre quella concezione eterodiretta ed organizzata che, da sempre, caratterizza i vari ambiti del turismo industriale (Amari, 2010).

Il Living Industry Tourism offre quindi al visitatore la possibilità di comprendere i processi e la cultura di imprese "simbolo" dei luoghi oggetto dell'esperienza turistica; inoltre alla conoscenza diretta di realtà produttive di eccellenza si affianca alla possibilità di testare direttamente i prodotti ed i "saperi" tipici.

Attraverso la valorizzazione dei patrimoni aziendali (know how, prodotti, brand, cultura d'impresa, ...) è possibile offrire una esperienza turistica innovativa e molto congruente con le aspettative di ampi segmenti di turisti "postmoderni".

Questa dimensione strategica dello sviluppo turistico della visita d'impresa appare particolarmente rilevante per due ragioni.

La prima è data dalla attuale proliferazione dell'offerta turistica che vede proporre sul mercato destinazioni e prodotti apparentemente "nuovi" ma, di fatto, caratterizzati da una forte omologazione. In questo senso, la possibilità di visitare imprese "vive", che rappresentano con vigore alcuni tratti peculiari delle culture locali, diviene un importante elemento di marketing territoriale.

La seconda ragione è che le imprese possono diventare una risorsa turistica innovativa anche (o forse soprattutto) per quei territori "non turistici" che solo di recente si stanno presentando come prodotti sul mercato della vacanza. In questo caso la visita d'impresa diviene una componente caratterizzante, in grado di attrarre

3 Living Industry Tourism può essere considerato un sinonimo di "Tourisme de la découverte économique", "Turismo de industria viva" e "Turismo d'impresa" etichette impiegate a vario titolo in alcuni Paesi europei per specificare meglio l'idea di turismo industriale. 
specifici segmenti di turisti altrimenti non raggiungibili da un'offerta più tradizionale o troppo "debole".

\section{LE IMPRESE COME PRODOTTO TURISTICO}

John Urry (1990) ha dimostrato con efficacia che il turismo si configura essenzialmente come un consumo visivo ed ha luogo quando uno "sguardo turistico" si posa su un oggetto. L'oggetto (manufatto, bene, servizio, ..., impresa) è in grado di attirare degli sguardi turistici solo nel caso in cui gli venga attribuita una qualche qualità turistica.

Un oggetto si trasforma quindi in attrazione quando è riconosciuto come tale dai consumatori-turisti. Il termine attrazione fa riferimento al rapporto tra un elemento oggettivo presente sul territorio ed i turisti che ne fanno uso. Affinché questo processo si inneschi, occorre che l'elemento oggettivo sia riconoscibile dai turisti e ne divenga fruibile; come afferma Dean MacCannell (1976), l'attrazione turistica può essere definita da "un sistema di relazioni tra tre elementi: un turista (l'elemento umano), un sight (l'elemento da osservare) ed un marker (l'elemento informativo). Un'attrazione turistica esiste quando i tre elementi sono connessi tra loro"4. Senza questa relazione il sight non è un'attrazione perché i turisti non lo riconoscono ed il loro sguardo non è in grado di decifrarlo.

Sulla base di questo modello, i marker assumono un peso sostanziale nell'offerta turistica di un luogo perché rappresenta il catalizzatore della trasformazione del sight in attrazione turistica offribile a vari segmenti di turisti.

In questo senso, la visita d'impresa può essere considerata tale per tre motivi principali: la scoperta di un tratto sicuramente autentico della back region, cioè il lavoro ed i suoi output; l'incontro con la comunità locale in una prospettiva innovativa nell'ambito delle relazioni host-guest, la possibilità di sperimentare con il test e l'acquisto il valore delle produzioni tipiche (Savoja, 2009a).

Naturalmente non si tratta di motivi tra loro esclusivi ed ognuno può essere declinato in vari modi; secondo l'aspetto che si vuole maggiormente sottolineare le visite possono essere molto diverse. Come, ad esempio le visite in situ che non necessitano di modifiche dei processi produttivi o del lay out organizzativo, le visite in situ che necessitano di strutture e personale aziendale ad hoc o le visite "show room" concepite come momento preliminare all'acquisto di prodotti presso punti vendita interni) ${ }^{5}$.

Tuttavia, al di là del format della visita, ciò che appare importante per comprendere la natura del turismo d'impresa è la motivazione aziendale soggiacente alla

4 D. MacCannell, The Tourist, pp. 41-42.

5 Le considerazioni e le informazioni riportate in questo paper provengono da un più ampio lavoro di ricerca svolto condotto nell'ambito dei lavori del $\mathrm{III}^{\circ}$ Congresso Europeo della visita d'impresa, Torino, Ottobre 2010; cfr. L. Savoja, La visita d'impresa come prodotto turistico. Una ricerca europea sui fattori di attrazione e le prospettive del turismo d'impresa, CCIIAA, Turin, 2010. 
scelta di "aprire le porte" ai visitatori. In effetti le imprese consentono le visite per motivi talvolta assai lontani da quelli turistici.

E' possibile individuare alcune macroaree per descrivere le principali motivazioni aziendali.

In questo caso il riferimento è all'analisi sistematica in relazione ai processi di scelta; si può fare riferimento al ciò che è stato definito come "approccio analitico" che si propone di evidenziare all'interno di un set di motivazioni tra loro complementari e gerarchicamente ordinate, quale sia la "motivazione prevalente" rispetto alla decisione considerata. Come si vedrà, il motivo prevalente che spinge le aziende ad organizzare visite ed ospitare degli estranei può essere ricondotto ad un obiettivo primario di "riconoscibilità", vale a dire l'interesse che le aziende hanno nel mostrarsi ad differenti categorie di stakeholder esterni.

Naturalmente l'idea di riconoscibilità è declinabile in vari modi, connessi alle dimensioni ed alla tipologia delle imprese; così, ad esempio, mentre le imprese commerciali di più piccole dimensioni vedono nella visita d'impresa uno strumento utile ad integrare il proprio marketing mix (riconoscibilità verso i clienti attuali e potenziali), quelle più grandi la considerano utile per rafforzare la propria immagine ed il proprio ruolo sociale rispetto a varie categorie di stakeholder come, ad esempio, i clienti attuali, la comunità locale, le istituzioni, ....

A partire da questo obiettivo primario è possibile individuare sei famiglie motivazionali.

IL “MOTIVO” PREVALENTE PER I COMPANY GUIDED TOUR

\begin{tabular}{|c|c|}
\hline COMPANY IMAGE & Visibilità dell'impresa \\
\hline & Visibilità del/dei marchi \\
\hline PROMOZIONALE & Comunicazione/Pubblicità di prodotto \\
\hline & Fidelizzazione \\
\hline COMMERCIALE & Vendita e commercializzazione di prodotti \\
\hline RESPONSABILITÀ & Affermazione del ruolo dell'azienda in quanto attore \\
territoriale
\end{tabular}


Si tratta evidentemente di tipologie non esclusive e tra loro complementari; in effetti le aree di sovrapposizione sono significative e per questo la classificazione proposta ha una valenza impressionistica.

Per Company Image si intende la volontà di affermare l'immagine positiva dell'impresa (o di marchi commerciali della stessa) agli occhi del pubblico. Questo obiettivo diviene ancor più specifico quando il gruppo di visitatori è composto da clienti già acquisiti, in questo caso la visita è un prezioso strumento per la loro fidelizzazione.

Così la visita motivata a migliorare la Company Image, pur non avendo degli specifici obiettivi promozionali o pubblicitari, diviene uno strumento delle strategie di marketing.

Più precisamente, si tratta di ciò che è definibile Corporate Identity; che va intesa come quell'insieme di tratti in grado di definire l'azienda sulla base di un repertorio di segnali e simboli familiari all'opinione pubblica.

Le motivazioni definite promozionali sono quelle più frequenti; in generale la visita ha una finalità promozionale quando si configura come uno strumento per comunicare al pubblico la forza di un marchio e/o la qualità dei prodotti; in questo caso ciò che assume rilievo è la possibilità di comunicare tali qualità anche attraverso la qualità della visita. Offrire la possibilità di visitare la propria impresa si configura quindi come una forma di pubblicità diretta.

Inoltre la pubblicità del prodotto mediante la visita è la motivazione fondamentale anche per quelle produzioni caratterizzate da forti connotati territoriali da affermare e diffondere.

Le motivazioni commerciali, vale a dire la visita intesa come strumento per la vendita diretta, sono contigue a quelle precedenti. Se nel caso del turismo dello shopping l'eventuale visita all'impresa è un momento facoltativo e propedeutico che ha la principale funzione di predisporre all'acquisto e, possibilmente, incrementarlo, nel caso del turismo d'impresa il criterio di importanza si capovolge e l'acquisto risulta essere un'attività accessoria alla visita ed all'acquisizione di nuove conoscenze.

Nel turismo dello shopping dunque la visita è facoltativa ed ancillare rispetto all'acquisto di merci, mentre nel turismo d'impresa l'acquisto è un'appendice, importante ma non indispensabile, dell'esperienza di visita. In questo caso la vendita diretta è importante soprattutto per caratterizzare la valenza turistica dell'esperienza di visita, visto che la dimensione commerciale è oggi sempre più significativa del "fare turismo".

Le altre motivazioni considerate nella tabella fanno riferimento a differenti dimensioni della riconoscibilità ricercata dalla aziende; si tratta dell'interesse a segnalare il proprio ruolo di "attore sociale".

E' un obiettivo riconducibile all'interno del più vasto ambito della Responsabilità Sociale d'Impresa (RSI) relativamente al quale il dibattito è assai ampio ed articolato e spazia da posizioni ultraliberiste che negano in maniera assoluta l'esistenza di altre responsabilità in capo alle aziende al di là di una legittima produzione di 
ricchezza a favore degli stockholder sino alle interpretazioni più ortodosse della cosiddetta teoria degli stakeholder che attribuiscono alle imprese una serie di "doveri" determinati dall'uso privatistico di beni collettivi non riproducibili.

Non è certo questo l'ambito per approfondire il discorso, tuttavia in estrema sintesi si può dire che le imprese, in quanto nodi di reticoli relazionali in grado di connotare e trasformare un territorio, hanno delle responsabilità nei confronti del territorio stesso; una forma per riconoscere l'esistenza di tali responsabilità può essere quella di accettare di ospitare dei visitatori e di organizzare delle visite per favorirne lo sviluppo turistico.

La visita come strumento della RSI assolve a due funzioni principali.

La prima è quella di rendere disponibile un maggior numero di informazioni al riguardo a disposizione degli stakeholder esterni; la seconda è quella di occupare un ruolo attivo come agente di promozione e sviluppo locale.

La visita diviene così una sorta di "servizio pubblico" a disposizione della collettività e ciò produce delle ricadute positive.

In primo luogo poter visitare un impresa è una opportunità per migliorare ed incrementare il network relazionale ed in definitiva il capitale sociale collettivo.

E' noto che tale incremento risulta essere un fattore decisivo per aumentare il benessere in un territorio ed anche il turismo (o meglio alcune forme di turismo) è un ambito di produzione di capitale sociale perché aumenta la circolazione di fiducia sistemica ed altri capitali relazionali (Álvarez Sousa, 2005). In quest'ottica, un'azienda aperta e "trasparente" è in grado di dialogare con profitto con gli altri attori locali e può adottare dei modelli di gestione proattivi più efficaci e vantaggiosi.

In secondo luogo la vista d'impresa va considerata come una risorsa collettiva in grado di arricchire il paniere delle ricchezze del territorio. Così, al di là della sua funzione economica, l'impresa diviene un asset specifico e rappresenta una componente attiva dell'offerta, anche turistica, di quei luoghi.

Un'altra tipologia di motivazioni riconducibile all'area della RSI è quella della funzione educativa assolta dalle visite d'impresa. Visitare un'impresa è uno strumento formativo ed è la base per lo sviluppo del turismo educativo che, pur poco analizzato e conosciuto, non può certo dirsi marginale.

\section{LA VISITA D'IMPRESA: CARATTERISTICHE ED ASPETTI ORGANIZ- ZATIVI}

Come si può facilmente immaginare, ogni visita rappresenta un unicum legato alle specificità di ogni realtà produttiva.

Al di là delle differenze tuttavia, è possibile individuare alcuni punti in comune. In primo luogo, le visite sono di norma arricchite da altre attività riconducibili a quattro ambiti: divulgazione, informazione, promozione e commerciale. 
A seconda che si tratti di un'azienda che produca per i mercati di massa o elabori prodotti rivolti a nicchie, la visita è orientata in maniera differente in modo da mettere al "centro della scena" di volta in volta quattro universi assiologici esplicitabili come: il prodotto e le sue caratteristiche di eccellenza; l'impresa e la sua cultura; i processi produttivi e le loro particolarità; il ruolo sociale dell'impresa. Sulla base dell'immagine che si vuole mettere maggiormente in luce, la visita assume tre forme distinte.

La prima è quella della "visita conoscitiva" durante la quale si vuole portare il visitatore a comprendere in maniera più approfondita rispetto alle sue conoscenze iniziali gli aspetti connessi ai prodotti ed ai processi produttivi. L'aspetto caratteristico di questa visita è informare il visitatore che alla fine dell'esperienza dovrà "saperne di più".

La seconda forma di visita è quella "promozionale-commerciale" che ha l'obiettivo di mettere in luce le qualità positive dei prodotti. Anche in questo caso le modalità per raggiungere tale scopo possono essere molteplici (dal test alla degustazione, dalla prova all'analisi delle caratteristiche salienti) e questa forma di visita si caratterizza per l'interesse a pubblicizzare e vendere i prodotti.

La "visita educativa" rappresenta la terza tipologia ed è orientata non solo alla semplice conoscenza dei prodotti e della produzione ma si trasforma in "viaggio" all'interno dell'impresa e della sua cultura fatta di valori, know-how, simboli e attività filantropiche sul territorio. In questo caso la visita favorisce la presa di coscienza del ruolo sociale svolto dall'impresa, l'avvicinamento dei visitatori ai tratti più profondi della cultura aziendale e, solo come conseguenza, alla loro trasposizione in buone prassi produttive e elevata qualità dei prodotti.

Tuttavia, al di là del fatto che la visita d'impresa si presenti come uno strumento promozionale o che si configuri come una vera e propria attività commerciale $\mathrm{o}$, ancora, che costituisca un'attività di "relazione", la sua struttura è riconducibile ad un modello generale descrivibile come un percorso di sei tappe che, pur potendo essere di importanza e durata diverse, devono essere presenti per definire la visita come forma di turismo d'impresa. 


\section{LE TAPPE DELLA VISITA D’IMPRESA}

\begin{tabular}{|c|c|c|c|}
\hline $\mathrm{I}^{\circ}$ & $\begin{array}{l}\text { ACCOGLIENZA } \\
\text { VISITATORI E } \\
\text { PRESENTAZIONE } \\
\text { DELL'IMPRESA }\end{array}$ & & \\
\hline $\mathrm{II}^{\circ}$ & $\begin{array}{l}\text { INTRODUZIONE } \\
\text { STORICA (impresa, } \\
\text { marchio, prodotto, ...) }\end{array}$ & + (eventuale) & $\begin{array}{c}\text { INFORMAZIONI TECNICHE } \\
\text { AGGIUNTIVE (processi } \\
\text { produttivi, oggetto del } \\
\text { business, caratteristiche delle } \\
\text { materie prime, ...) }\end{array}$ \\
\hline III $^{\circ}$ & PERCORSO DI VISITA & & \\
\hline $\mathrm{IV}^{\circ}$ & $\begin{array}{c}\text { CONOSCENZA } \\
\text { DEL PRODOTTO, } \\
\text { DEL PROCESSO } \\
\text { (degustazioni, test di } \\
\text { prodotto, informazioni } \\
\text { aggiuntive, dimostrazioni } \\
\text { pratiche, analisi sensoriali, } \\
\ldots \text {..) }\end{array}$ & & \\
\hline $\mathrm{V}^{\circ}$ & $\begin{array}{c}\text { ATTIVITÀ } \\
\text { ACCESSORIE } \\
\text { (fornitura di omaggi, } \\
\text { merchandising di } \\
\text { oggettistica e gadget } \\
\text { aziendali, informazioni } \\
\text { commerciali, ...) }\end{array}$ & + (eventuale) & $\begin{array}{l}\text { COMMERCIALIZZAZIONE } \\
\text { (vendita diretta, visita ed } \\
\text { acquisto a spacci aziendali e } \\
\text { punti vendita interni, ...) }\end{array}$ \\
\hline $\mathrm{VI}^{\circ}$ & COMMIATO & + (eventuale) & $\begin{array}{c}\text { FEED BACK } \\
\text { (questionario di valutazione, } \\
\text { verifica informale con i } \\
\text { visitatori }\end{array}$ \\
\hline
\end{tabular}

Il momento topico è il percorso di visita (tappa $\mathrm{III}^{\circ}$ ) ma la visita non può dirsi completa se manca una serie di attività complementari. In altre parole si può parlare di turismo d'impresa solo se esiste almeno un momento in cui il turista-visitatore percorre un tragitto all'interno dell'impresa osservando direttamente il processo produttivo (o alcune sue fasi); tuttavia la visita d'impresa può dirsi tale solo se vi è anche 
la possibilità di approfondire le conoscenze in merito ai vari aspetti delle lavorazioni o alle caratteristiche dei prodotti, di testarli e provarli e di conoscere i dettagli tecnici e commerciali. A tutto ciò si aggiungono i momenti di benvenuto e di commiato durante i quali, di norma, vengono forniti dettagli in merito alla storia dell'azienda.

La tabella che segue collega le due classificazioni proposte in precedenza considerando congiuntamente le motivazioni aziendali e le scelte organizzative.

\section{LE ATTIVITA' ACCESSORIE ALLA VISITA D'IMPRESA IN BASE ALLE MOTIVAZIONI AZIENDALI}

\begin{tabular}{|c|c|c|}
\hline $\begin{array}{l}\text { Motivazioni aziendali } \\
\text { alle visita }\end{array}$ & $\begin{array}{l}\text { Attività accessorie } \\
\text { in generale }\end{array}$ & $\begin{array}{l}\text { Commercializzazione dei prodotti } \\
\text { (varie forme di vendita diretta) }\end{array}$ \\
\hline COMPANY IMAGE & $\begin{array}{l}\text { Si, non sempre } \\
\text { caratterizzanti la } \\
\text { visita }\end{array}$ & $\begin{array}{l}\text { Non abituale, talvolta } \\
\text { merchandising di prodotti } \\
\text { accessori }\end{array}$ \\
\hline PROMOZIONALE & $\begin{array}{l}\text { Si, normalmente } \\
\text { caratterizzanti la } \\
\text { visita }\end{array}$ & $\begin{array}{l}\text { Non sempre presente, } \\
\text { merchandising di prodotti } \\
\text { accessori, possibilità di acquisto } \\
\text { nei punti vendita aziendali talvolta } \\
\text { a condizioni di favore }\end{array}$ \\
\hline COMMERCIALE & $\begin{array}{l}\text { Si, finalizzate alla } \\
\text { vendita }\end{array}$ & $\mathrm{Si}$, caratterizza la visita \\
\hline $\begin{array}{l}\text { RESPONSABILITÀ } \\
\text { SOCIALE }\end{array}$ & $\begin{array}{l}\text { Si, non sempre } \\
\text { caratterizzanti la } \\
\text { visita }\end{array}$ & Non abituale \\
\hline FORMAZIONE & $\begin{array}{l}\mathrm{Si}, \text { normalmente } \\
\text { caratterizzanti la } \\
\text { visita }\end{array}$ & $\begin{array}{l}\text { Non abituale, possibilità di } \\
\text { acquisto nei punti vendita aziendali } \\
\text { talvolta a condizioni di favore }\end{array}$ \\
\hline
\end{tabular}


Come si può notare, le tappe $\mathrm{IV}^{\circ} \mathrm{e} \mathrm{V}^{\circ}$ del percorso "tipo" costituiscono gli ambiti dove le differenze appaiono più marcate.

Naturalmente, la tabella generalizza situazioni molto diverse tra loro, tuttavia si può concludere che la "semplice" attività di vista non soddisfa i requisiti necessari per il turismo d'impresa anche perché molte attività "accessorie" sono considerate come fondamentali dai visitatori (Di Iorio et al., 2006).

\section{LA VISITA D'IMPRESA: I FATTORI DI ATTRAZIONE TURISTICA}

La capacità di attrazione turistica della visita d'impresa emerge riguardo a due dimensioni principali.

La prima è riferita alle potenzialità turistiche delle industrie vive mentre la seconda è relativa alla capacità autonoma che il turismo d'impresa può avere per strutturasi come prodotto turistico "in sé", sulla base dell'appeal delle imprese che organizzano le visite. In questo secondo ambito la visita d'impresa può svolgere un ruolo importante in territorio o città industriali che si aprono progressivamente al turismo in quanto elemento fondante e fruibile dell'identità locale.

I principali plus turistici della visita d'impresa possono essere ricondotti a 7 punti principali.

1. Diversifica e differenzia; in quanto rende più ricca l'offerta turistica ("più cose da fare") ma, soprattutto, la rende differente da altre offerte sempre più simili e standardizzate (in effetti, le imprese sono dei veri e propri unicum difficilmente omologabili).

2. Non è stagionale; normalmente è possibile visitare le imprese sempre durante tutto il corso dell'anno.

3. Possiede connotati innovativi; perché permette di vivere un'esperienza diretta dentro un luogo vivo e non sterilizzata sulla base delle esigenze dei produttori di turismo.

La sperimentazione diretta, le prove, gli assaggi, soddisfano quella "voglia di fare" congruente alla ricerca di "spendere bene" il proprio tempo libero che è uno dei traguardi più importanti per il turista contemporaneo.

4. Porta a visitare luoghi e territori turisticamente diversi e a tracciare nuovi itinerari turistici in zone dove il turismo è già presente.

Il tessuto industriale per molto tempo è stato considerato un disvalore e per questo gli itinerari turistici più classici di norma ignoravano interi territori che ora, anche grazie al turismo industriale, divengono interessanti per segmenti sempre più ampi di visitatori.

5. E' un'esperienza autentica; vistare un impresa viva è, più di altre attività turistiche, un modo per conoscere direttamente una dimensione originale dei luoghi visitati e soddisfare la ricerca dell'autenticità. 
6. Permette un contatto privilegiato con la back region e con la cultura locale; in quanto favorisce una conoscenza non mediata delle produzioni tipiche.

E' noto infatti che 1'"appropriazione" del tipico è un must del turista contemporaneo (Savoja, 2009b).

7. E' un'attività poco costosa.

Quando la visita non è addirittura gratuita, i costi diretti che i visitatori devono sostenere sono assai ridotti, soprattutto se paragonati a quelli sostenuti per fruire di altre attrazioni turistiche (musei, esposizioni, eventi, parchi a tema, ...).

Ovviamente la capacità di offrire le visite d'impresa come prodotti turistici corrisponde al saper far emergere questi plus.

Inoltre, l'aumento delle opportunità di visita fa crescere il valore dell'offerta turistica locale; tale aumento si traduce anche in maggiori opportunità per la comunità locale (basti pensare al ruolo didattico e formativo delle visite ad imprese) rappresentando così un incremento di quello che si può definire "capitale sociale" e, in definitiva, un miglioramento della qualità della vita per il territorio in questione e le popolazioni che lo frequentano.

In definitiva bisogna "pensare" alle visite d'impresa come bene collettivo di interesse per il turismo rispetto al quale è necessario il coinvolgimento attivo di tutti gli attori (Beritelli, 2011). Tale coinvolgimento, come si discuterà nel paragrafo successivo, pare il prerequisito necessario per l'affermazione della visita d'impresa come prassi del vivere contemporaneo.

\section{COME OFFRIRE AI TURISTI IL “PRODOTTO” VISITA D'IMPRESA?}

Si tratta di una questione evidentemente rilevante perché occorre affrontare questioni strategiche e problemi pratici di vario genere.

Tra i più rilevanti vi è la difficoltosa percezione del valore turistico della visita che normalmente si configura come attività time consuming poco funzionale alle disponibilità in termini di tempo (oltre ad occupare molto tempo, si svolgono in orari turisticamente poco propizi) e mobilità (la visita sovente impone spostamenti in zone lontane dai percorsi turistici più consolidati) del turista medio.

Un'altra questione è che l'incontro "spontaneo" tra domanda e offerta è arduo a causa della scarsa connotazione ludica del turismo d'impresa e della sua ridotta integrazione con le altre attrazioni.

In effetti le industrie, i siti di produzione, le fabbriche e gli uffici non sono luoghi pensati, progettati ed organizzati per ospitare dei visitatori esterni. In questo senso, la presenza di estranei è una condizione particolare in grado di influenzare vari aspetti legati alla produzione ed alla distribuzione.

I problemi pratici più rilevanti possono essere raggruppati in cinque categorie: la salute e sicurezza dei visitatori, l'igiene dei luoghi di lavoro, la sicurezza, 
l'organizzazione della produzione e la riservatezza. La tabella che segue illustra gli aspetti che limitano le possibilità di vista ed alcune soluzioni adottate per superare tali criticità.

\section{LE CRITICITA’ DELLA VISITA D'IMPRESA NELL'OTTICA DELLE AZIENDE}

\begin{tabular}{|c|c|c|}
\hline & Aspetti Limitanti & Alcune Soluzioni Possibili \\
\hline \multirow[t]{2}{*}{$\begin{array}{l}\text { SICUREZZA E } \\
\text { SALUTE DEI } \\
\text { VISITATORI }\end{array}$} & Rischio di infortuni & $\begin{array}{l}\text { Creazione di percorsi di visita obbligati, Utilizzo di veicoli } \\
\text { riservati, Schermatura e protezioni fisiche di tratti del percorso } \\
\text { di visita, Visite vietate ai minori (talvolta under 16) }\end{array}$ \\
\hline & Rischio di allergie & $\begin{array}{l}\text { Esclusione dal percorso di visita di aree produttive, Visite } \\
\text { organizzate quando non vengono impiegate materie prime } \\
\text { potenzialmente allergizzanti, Visite vietate ai minori }\end{array}$ \\
\hline \multirow[t]{2}{*}{ IGIENE } & $\begin{array}{l}\text { Salubrità } \\
\text { dell'ambiente di } \\
\text { lavoro }\end{array}$ & $\begin{array}{l}\text { Obbligo per i visitatori di indossare indumenti e calzature } \\
\text { idonee fornite dall'impresa per la visita }\end{array}$ \\
\hline & $\begin{array}{c}\text { Rischio di } \\
\text { contaminazione dei } \\
\text { prodotti }\end{array}$ & $\begin{array}{c}\text { Esclusione dal percorso di visita di alcune fasi produttive e/o } \\
\text { lavorazioni }\end{array}$ \\
\hline \multirow[t]{2}{*}{$\begin{array}{c}\text { SICUREZZA } \\
\text { DELL'AZIENDA }\end{array}$} & $\begin{array}{l}\text { Identità personale dei } \\
\text { visitatori }\end{array}$ & $\begin{array}{l}\text { Richiesta di elenco nominativo dei visitatori con fotocopia } \\
\text { di un documento di identità, Controllo dell'identità all'inizio } \\
\text { della visita, Visite limitate a gruppi precostituiti, Visite } \\
\text { individuali non autorizzate, Visite vietate ai minori }\end{array}$ \\
\hline & Rischio di furti & $\begin{array}{l}\text { Controllo durante la visita, Controllo al termine del percorso } \\
\text { di visita, Visite vietate a gruppi scolastici o troppo numerosi }\end{array}$ \\
\hline \multirow[t]{4}{*}{$\begin{array}{l}\text { ORGANIZZAZIONE } \\
\text { DELLA } \\
\text { PRODUZIONE }\end{array}$} & $\begin{array}{l}\text { Visite possibili solo in } \\
\text { determinati momenti } \\
\text { della giornata e/o } \\
\text { dell'anno }\end{array}$ & Limitazione delle visite a determinati periodi \\
\hline & $\begin{array}{l}\text { Visite possibili in } \\
\text { base allo stato dei } \\
\text { lavori per specifiche } \\
\text { commesse }\end{array}$ & $\begin{array}{l}\text { Limitazione delle visite in base allo stato di avanzamento dei } \\
\text { lavori }\end{array}$ \\
\hline & $\begin{array}{c}\text { Reparti con } \\
\text { lavorazioni } \\
\text { potenzialmente } \\
\text { rischiose (materiali, } \\
\text { rumorosità, } \\
\text { temperatura ...) } \\
\end{array}$ & $\begin{array}{l}\text { Organizzazione di visite che escludono reparti critici e/o } \\
\text { potenzialmente pericolosi, Visite vietate ai minori }\end{array}$ \\
\hline & $\begin{array}{l}\text { Interferenze con i } \\
\text { macchinari }\end{array}$ & Divieto di usare i telefoni cellulari \\
\hline RISERVATEZZA & $\begin{array}{l}\text { Rischio di spionaggio } \\
\text { industriale }\end{array}$ & $\begin{array}{c}\text { Controllo durante la visita, divieto di fotografare, registrare o } \\
\text { filmare durante la visita, Divieto di usare i telefoni cellulari, } \\
\text { Organizzazione di percorsi di visita che escludono reparti } \\
\text { critici e/o aree sensibili }\end{array}$ \\
\hline
\end{tabular}


Esistono anche inibitori esterni alle aziende che sono in grado di ostacolare le visite d'impresa come ad esempio la difficoltà a raggiungere con i mezzi pubblici i siti produttivi da visitare o quelle connesse alla richiesta di visita, tuttavia per le aziende oggetto di interesse turistico, l'aspetto fondamentale è la possibilità di programmare le visite in maniera coerente con i processi organizzativi. Questa necessità è sovente in contrasto con quelle degli "organizzatori turistici” ed il contrasto può determinare delle incongruenze e rendendo l'offerta di turismo d'impresa meno appetibile. Le incongruenze riguardano la flessibilità (intesa come maggiore libertà di scelta di tempi e modi della visita), la rapidità (intesa come possibilità di decidere l'effettuazione della visita anche d'impulso) e la varietà (intesa come disponibilità di un ventaglio ampio di opportunità sia come date disponibili per la visita ad una stessa azienda sia come diversificazione delle aziende da visitare) così come si è tentato di specificare nella tabella che segue.

\section{PRINCIPALI PROBLEMI NELLA STRUTTURAZIONE DELLA VISITA D'IMPRESA COME PRODOTTO TURISTICO}

\begin{tabular}{|c|c|}
\hline COMUNICAZIONE & $\begin{array}{l}\text { Contattare le aziende non è facile e non sempre è evidente } \\
\text { chi è il referente }\end{array}$ \\
\hline & $\begin{array}{l}\text { Talvolta le aziende non desiderano la mediazione di } \\
\text { intermediari commerciali nell'organizzazione della visita }\end{array}$ \\
\hline & $\begin{array}{c}\text { Il lasso di tempo per ottenere una risposta alle richieste } \\
\text { di visita è eccessivo e non permette una adeguata } \\
\text { programmazione turistica }\end{array}$ \\
\hline \multirow[t]{3}{*}{$\begin{array}{l}\text { CADENZA E } \\
\text { FREQUENZA DELLE } \\
\text { VISITE }\end{array}$} & $\begin{array}{l}\text { La disponibilità delle aziende è generalmente troppo ridotta } \\
\text { per poter inserire stabilmente la visita nel portafoglio prodotti } \\
\text { o anche solo per promuoverla }\end{array}$ \\
\hline & $\begin{array}{l}\text { I vincoli in termini di orario e giorni riservati alle visite } \\
\text { sovente rappresentano un ostacolo che esclude a priori molti } \\
\text { segmenti di turisti interessanti e potenzialmente interessati }\end{array}$ \\
\hline & $\begin{array}{c}\text { In generale le aziende autorizzano un numero troppo esiguo } \\
\text { di visite che rende poco interessante ogni investimento } \\
\text { promozionale }\end{array}$ \\
\hline \multirow[t]{2}{*}{$\begin{array}{l}\text { IDENTITÀ E } \\
\text { CARATTERISTICHE } \\
\text { DEI VISITATORI }\end{array}$} & $\begin{array}{c}\text { La (giusta) richiesta da parte delle aziende di conoscere } \\
\text { con anticipo l'esatto numero e l'identità personale dei } \\
\text { visitatori sovente è incompatibile con le prassi turistiche più } \\
\text { consolidate }\end{array}$ \\
\hline & $\begin{array}{c}\text { Le limitazioni in termini di numero, età ed altre } \\
\text { caratteristiche dei visitatori limitano la possibilità di offrire la } \\
\text { visita a specifiche categorie di turisti }\end{array}$ \\
\hline
\end{tabular}


GESTIONE DEGLI IMPREVISTI
Le aziende sono poco flessibili nella gestione degli imprevisti, peraltro strutturali nel business turistico

Oltre alle problematiche organizzative descritte, va rilevata anche l'esistenza di una criticità di rilievo collegata alla "catena del valore" della visita d'impresa; in altre parole la definizione dei meccanismi distributivi costi-benefici di questa ipotetica filiera di prodotto turistico.

A ben vedere, nel caso del turismo d'impresa, la definizione costi-benefici non sempre trova delle risposte soddisfacenti all'interno della classica relazione clientefornitore e, pur in una prospettiva particolare, richiama questioni tipiche dei modelli multistakeholder di gestione d'impresa.

Se l'ipotesi è l'aumento delle possibilità di visita presso le aziende attraverso la loro commercializzazione sul mercato turistico aumentando così i visitatori di tutto il territorio di riferimento, di fatto si riconosce alle imprese stesse il "ruolo" di prodotto turistico.

Da tale riconoscimento consegue l'attribuzione alle stesse del connotato di bene collettivo ed il suo impiego non può essere ricondotto tout court nell'alveo delle relazioni di mercato.

L'incremento degli arrivi e delle presenze turistiche produce dei benefici diretti per i vari operatori del settore ed all'indotto turistico; se tali benefici sono prodotti dalla possibilità di visitare, così come si fa per altre attrazioni, le imprese del territorio, va definito il modo di distribuire i "costi" ed i "benefici" generati dalla maggiore presenza di visitatori in quel territorio.

E' già stato rilevato che esistono casi in cui tale suddivisione è evidente e la catena del valore è ben definita; tuttavia non sempre è così.

Alcune aziende non hanno interessi commerciali diretto che aumentano proporzionalmente all'aumentare del numero di visitatori. La questione non è certo marginale, sovente infatti si trovano in questa situazione proprio le aziende "icone" del territorio (e quindi più dotate di reale appeal turistico).

In questi casi non è certo possibile immaginare delle relazioni tra organizzatori turistici ed aziende da visitare strutturate secondo il modello cliente-fornitore. La catena del valore non esiste; l'aumento dei visitatori, reso possibile dall'opera degli intermediari turistici (opera che per sua natura è immaginabile solo se ha finalità commerciali orientate alla produzione di profitto), genera un aumento di costi a carico delle imprese visitate; aumenti che in prospettiva aziendale, non sempre risultano giustificabili.

Laddove il rapporto tra le aziende da visitare e gli operatori turistici non è riconducibile al modello fornitore-cliente (o a quello del co-marketing), le Istituzioni potrebbero svolgere un ruolo importante. Si tratta della capacità di creare delle forme di "remunerazione indiretta" interessanti; in altre parole occorre trovare il modo per ricompensare il "lavoro di ospitalità turistica" svolto dalle aziende.

Il discorso è generico e, ovviamente, andrebbe approfondito in maniera ben più precisa di quanto proposto in queste note. Tuttavia, visto che il valore turistico di 
un'azienda non può essere confinato tra i suoi asset privati, è indubbio che il suo impiego produce dei benefici che ricadono anche su altri attori. In questo senso dunque, la presenza di visitatori in una azienda è certamente positiva per l'azienda stessa ma, altrettanto certamente, è positiva anche per la collettività intesa nel senso più ampio (dipendenti, operatori del settore turistico, sistema locale, ...).

Da questa constatazione, difficilmente opinabile, discende allora che le aziende che decidono di "aprire le porte" a visitatori e turisti creano benessere a favore di tutto il territorio e quindi vanno incentivate a farlo di più ed in maniera più funzionale alle esigenze dei visitatori.

Così, in maniera assolutamente esemplificativa, si può pensare ad incentivi di vario genere (contributivi, fiscali, connessi alla gestione delle risorse umane, alla formazione, ...) che possono essere determinati in base alla tipologia dell'azienda che offre la visita, al numero di visitatori, alle loro caratteristiche, alla congruenza tra la disponibilità dell'azienda e le necessità dei visitatori-turisti o ad altri parametri più specifici.

In definitiva si può affermare che lo sviluppo del Living Industry Tourism è legato alla capacità di rendere compatibili gli interessi degli attori coinvolti.

Interessi che, pur non contrapposti, non sono ancora complementari.

\section{TURISMO D'IMPRESA. IL CASO "MADE IN TORINO"“}

L'iniziativa "Made in Torino - Tour the Excellent" nasce nel 2005 per volontà della Camera di Commercio di Torino in collaborazione con Turismo Torino (si tratta della locale Agenzia di accoglienza turistica) e con il supporto della Provincia di Torino con l'obiettivo di impiegare, per la promozione della città e per completare il prodotto turistico territoriale, uno dei tratti distintivi di questa grande città. Come noto, il capoluogo piemontese è una delle città europee maggiormente caratterizzate da una marcata identità industriale e dalla presenza di eccellenze produttive a livello mondiale.

La sua essenza di capitale industriale è consolidata a partire dall'esteso patrimonio infrastrutturale frutto dell'epopea della rivoluzione industriale che ha trovato in Italia il suo apice proprio a Torino (AA.VV, 1999). Oggi Torino, pur continuando a mantenere una struttura essenzialmente industriale, sta vivendo ulteriori trasformazioni e la One Company Town degli anni 80- caratterizzata dalla presenza delle fabbriche della Fiat- è oggi una moderna metropoli dove alle eccellenze industriali si affianca un forte dinamismo imprenditoriale nel comparto del terziario avanzato ed un rilevante aumento delle presenze turistiche, soprattutto a partire dal grande successo delle Olimpiadi invernali del 2006.

6 Il paragrafo contiene una versione ridotta del paper Savoja L., Coss F., Company Guided Tours as Tourist Attractions. A case of success: Made in Torsión-Tour the Excellent, presentato al seminario UNWTO "Industrial Heritage in Tourism Policies for Sustainable Development” Zabrze - Poland, April 2011. 
In questo senso "Made in Torino", tenendo conto del sempre maggiore peso che il turismo industriale occupa nei modelli contemporanei di consumo del tempo libero, si configura come un'iniziativa che esalta l'imprinting di città industriale di cui Torino è dotata e lo trasforma in un punto di forza per aumentare la sua attrattività turistica.

Le visite d'impresa offerte da "Made in Torino" sono pensate, promosse ed offerte ai turisti individuali allo stesso modo delle altre attrazioni più classiche (musei, eventi, concerti, scenari, shopping, prodotti tipici, ....) presenti in città.

Torino si afferma così come la prima destinazione della vista d'impresa in Italia in grado di includere in un'unica iniziativa integrata, un ampio ventaglio di prestigiose eccellenze industriali e imprenditoriali, simbolo del Made in Italy nel mondo ${ }^{7}$.

L'innovatività dell'iniziativa è rilevabile in particolare in due ambiti distinti.

Il primo è la considerazione della visita d'impresa in quanto attrazione con le caratteristiche di prodotto "vendibile" all'indistinto pubblico dei turisti individuali; il secondo è il processo di integrazione con le altre componenti del prodotto turistico territoriale.

Il modello di funzionamento si basa sulla presenza congiunta di tre attori: $\mathrm{Ca}-$ mera di Commercio di Torino (Organo decisore, Board di controllo, Finanziatore); Turismo Torino e Provincia (Organizzatore, Promotore e Responsabile del funzionamento per le fasi di prenotazione, trasporto da e per le aziende, valutazione della soddisfazione, aggiornamento dell'offerta, gestione dei calendari di visita); Aziende (Autorizzano visite in date prefissate riservate ai turisti veicolati da Turismo Torino e Provincia e gestiscono le fasi di visita e la presenza dei visitatori al loro interno).

Il ruolo dei tre attori è definito secondo lo schema organizzativo sintetizzato nella tabella successiva.

\begin{tabular}{|c|c|}
\hline Camera di Commercio di Torino & $\begin{array}{c}\text {-sensibilizzare le aziende ad aprire le porte ai } \\
\text { visitatori }\end{array}$ \\
$\begin{array}{c}\text {-assicurare la fattibilità economica e la copertura } \\
\text { finanziaria dell'iniziativa }\end{array}$ \\
Turismo Torino e Provincia & $\begin{array}{c}\text {-definire e organizzare le attività } \\
\text {-delineare, gestire e prenotare le visite } \\
\text {-sviluppare il prodotto }\end{array}$ \\
\hline Aziende partecipanti & $\begin{array}{c}\text {-promozione e marketing dell'iniziativa } \\
\text { - gestire le visite ed organizzarle sulla base di un } \\
\text { criterio omogeneo }\end{array}$ \\
\hline
\end{tabular}

7 Tra le 25 aziende torinesi che, ad oggi, partecipano all'iniziativa si possono ricordare Alenia, Aurora, Fiat, Iveco, Lavazza, Martini \& Rossi, Thales solo per citare i nomi più noti. 
L'idea che si vuole trasmettere è che "Made in Torino" non offre "semplici visite a semplici fabbriche", ma vere e proprie scoperte delle aziende, del loro know how, dei loro prodotti e del loro collegamento con il territorio nel quale opera.

Per questo, le oltre 100 visite che nel 2011 compongono il prodotto "Made in Torino", sono state raggruppate all'interno di cinque filiere tematiche che raggruppano le aziende partecipanti sulla base di un immagine che richiama le peculiarità del settore secondario torinese. Si tratta delle filiere Automotive e design, Aerospazio, Alimentare, Editoria e stampa, Oggettistica e gioielleria.

\section{IL TURISMO D'IMPRESA A TORINO DAL 2005 AD OGGI.}

\section{- L'ORGANIZZAZIONE DELLE VISITE}

Nel 2005 le prime aziende ad aderire al progetto "Made in Torino-Tour The Excellent" sono state quelle dei settori automotive, design e dell'oggettistica di lusso, in seguito, nel corso degli anni il numero delle imprese partecipanti si è notevolmente incrementato, includendo via via alcune aziende leader mondiali del settore aerospaziale fino a completare l'offerta con il settore agroalimentare ed enologico; infine, nel 2011 si registra l'ingresso della filiera della stampa e dell'editoria.

Oggi, per i turisti individuali di Torino vi è la possibilità di vistare ben 25 aziende riconducibili alle varie filiere tematiche. I posti disponibili per ogni visita oscillano tra le 15 e le 25 unità.

Le visite sono state studiate in particolare per turisti autonomi, principalmente "short break" e si svolgono il venerdì, il sabato ed il lunedì con partenza dal centro città verso i vari siti produttivi. Esse hanno una durata di 3-4 ore complessive e gli spostamenti da e per le imprese si effettuano a bordo di un bus, con l'accompagnamento di una guida di Turismo Torino e Provincia che rimane insieme al gruppo per tutta la durata del tour. I percorsi all'interno delle aziende sono a cura del personale delle aziende stesse ed avvengono in lingua italiana. Talvolta, a seconda della presenza di turisti stranieri sono previsti dei tour in lingua inglese o francese.

Il costo varia da $€ 10,00$ a di $€ 5,00$ a persona e comprende, oltre al trasporto anche la copertura assicurativa per eventuali infortuni ${ }^{8}$.

\section{- I RISULTATI DELL'INIZIATIVA}

L'idea di declinare in un binomio "stretto" industria e turismo è stata coraggiosa ma si è rivelata vincente da diversi punti di vista. I risultati di "Made in Torino" sono positivi e testimoniano, oltre al successo dell'iniziativa presso il pubblico di riferimento, anche le grandi potenzialità del turismo d'impresa.

La crescita è stata continua; si è passati da 9 aziende partecipanti nel 2005 a 25 nel

8 Maggiori informazioni su www.turismotorino.org. Con l'idea di dare maggiore visibilità alla dimensione turistica della visita sono creati e resi disponibili anche degli spazi virtuali per lo scambio di immagini ed opinioni cfr. www.youtube.com/TurismoTorino e www.flickr.com/photos/turismotorino. 
2011 per un totale di 104 visite programmate per il 2011 e con una previsione di ulteriori allargamenti per il biennio successivo.

Nel primo semestre del 2011 l'incremento dei visitatori è del 46\% rispetto allo stesso periodo dell'anno precedente e nel secondo semestre, in base alle stime sulle visite già effettuate e le prenotazioni, è destinato a salire sino al 57\% sino a superare il numero di 1200 turisti individuali che in questo anno avranno visitato un impresa con "Made in Torino".

Nel caso di questo prodotto, non è tanto importante rilevare la dimensione assoluta dei visitatori veicolati (è evidente che i turisti individuali sono una minoranza rispetto ai visitatori organizzati in gruppi professionali, scolastici, provenienti dal turismo MICE, ... ${ }^{9}$ ) quanto piuttosto l'elevato tasso di riempimento dei posti riservati dalle aziende ai "clienti" di TurismoTorino.

Il load factor relativo a tutte le visite calendarizzate è costante e presenta un medio del $85 \%$ con un massimo di $90 \%$ nel 2008 .

Si è trattato di visitatori che provengono per il 75\% circa dei casi da fuori città (con un $20 \%$ di turisti non italiani).

\section{QUALE FUTURO PER IL TURISMO D'IMPRESA?}

Al di là delle sue indubbie potenzialità e del sempre più evidente sviluppo, la visita d'impresa, pur facendo già parte delle abitudini turistiche di numerosi individui, non è ancora una parte integrante del "prodotto turistico" che i visitatori percepiscono e come tale consumano.

La parziale integrazione della visita d'impresa con gli altri elementi che compongono un prodotto turistico globale acuisce la distanza tra tale attività e la sua percezione in quanto attrazione destinata ad un indistinto pubblico di turisti, mentre il suo interesse per segmenti specifici è consolidato.

Per questo continua ad esistere la difficoltà di attribuire all'esperienza di visita un significato compiutamente turistico; per questo la differenza esistente tra ciò che viene "venduto" e ciò che viene "acquistato", tra ciò che viene "acquistato" e ciò che viene "consumato" e, infine, tra ciò che viene "consumato" e ciò che viene "valutato", permane.

Tutto ciò significa che il consumatore-turista non può razionalizzare completamente la relazione esistente tra il bene-servizio fruito e l'insieme della sua esperienza turistica.

Tali difficoltà paiono rilevanti soprattutto per quelle attività, come la visita d'impresa, che non possiedono specifiche valenze ludiche o di leisure né possiedono i connotati dell'attrazione turistica classica.

9 Il turismo d'impresa di tipo organizzato a Torino è una pratica assai sviluppata; basti pensare agli oltre 23000 visitatori che ogni anno varcano i cancelli della fabbrica Mirafiori della Fiat o ai quasi 8000 che visitano gli stabilimenti di Pessione della Martini \& Rossi 
Pare configurarsi una contraddizione di fondo che occorrerà analizzare con attenzione. Si tratta del carattere duale della visita d'impresa determinato, da una parte dalla sua parziale estraneità dall'universo turistico e dall'altra dalla sua, relativa, diffusione tra le pratiche turistiche contemporanee.

Tale dualismo risulta assai poco congruente con le chanche di sviluppo di questa pratica turistica.

Riprendendo la classica distinzione delle motivazioni che stanno alla base delle scelte turistiche che distingue tra push e pull motivation (Crompton, 1979) si può ipotizzare che visitare un'impresa abbia ridotte capacità motivazionali in entrambi i casi.

In primo luogo perché non è possibile far rientrare la visita d'impresa nel set di attività capaci di soddisfare motivazioni di tipo push che spingono l'individuo lontano dalla routine e sostengono il suo desiderio di "abbandonare qualcosa" (frequentemente il desiderio di fare turismo si esprime attraverso la volontà di abbandonare il lavoro, la quotidianità, gli impegni professionali, ... vale a dire tutti aspetti "concettualmente" vicini all'impresa) spingendo al consumo di un prodotto turistico al di là delle caratteristiche del prodotto stesso.

In secondo luogo perché, oggi, la visita d'impresa non pare possedere ancora l'appeal turistico sufficiente per soddisfare motivazioni di tipo pull che attirano il turista e lo spingono a raggiungere delle mete specifiche.

Le motivazioni pull che tirano il turista verso il prodotto (verso l'attività, verso l'attrazione) traggono il loro significato dai connotati dell'offerta che deve essere turisticamente "comprensibile" ed in grado di far capire ai potenziali utenti il suo valore turistico.

Oggi queste capacità paiono essere ancora inespresse nel Living Industry Tourism, anche se, indubbiamente in questo ambito i margini di crescita sono ampi ed assai promettenti.

Le due tipologie rientranti nelle pull motivation (sperimentare delle novità ed aumentare le proprie conoscenze) stanno diventando sempre più importanti per $\mathrm{i}$ turisti e probabilmente il "futuro turistico" della visita d'impresa si giocare proprio in questo ambito. Per molto tempo l'offerta turistica ha rivolto una attenzione quasi esclusiva alle push motivation dei consumatori mentre non ha fatto lo stesso per le pull motivation, mentre è proprio rispetto a queste ultime, che esistono interessanti spazi che il turismo d'impresa potrebbe occupare con successo.

\section{RIFERIMENTI BIBLIOGRAFICI}

AA.VV. (1999): 100 luoghi dell'industria a Torino e in Piemonte, Associazione Torino Città Europea, Torino.

Álvarez Sousa, A. (2005): La contribución del turismo al desarrollo integral de las sociedades receptoras. Aspectos teóricos-metodológicos, Política y Sociedad, $42(1)$. 
Amari, M. (2010): L'industria sposa il turismo, La Rivista del Turismo-Touring Club Italiano, vol. 3.

Beritelli, P. (2011): Cooperation among Prominent Actors in a Tourist Destination, Annals of Tourism Research, 38 (2).

Crompton, J. (1979): Motivation for Pleasure Vacation, Annals of Tourism Research, $6(4), 1979$

Di Iorio, V. et al. (2006): Marketing per il turismo d'impresa, PromoVeneto, Vicenza. Farrell B.; Twining-Ward, L. (2004), Reconceptualizing Tourism, Annals of Tourism Research, 31 (2).

Giddens, A. (2000): The Third Way and Its Critics, Polity Press, Cambridge.

Jansen-Verbeke, M. (1998): Tourismification of Historical Cities, Annals of Tourism Research, 25 (3).

MacCannell, D. (1976): The Tourist, Schocken Books, New York.

Morice, J-R. (2006): La visite d'entreprise en Europe. Un champ à explorer, Cahier Espace, vol. 92.

Savoja, L. (2009): L'identità locale come fattore di successo turistico, Aracne, Roma.

Savoja, L. (2009): Lo típico como recurso turístico entre sostenibilidad social y participación popular, in M. Latiesa Rodríguez, I. Puertas Cañaveral, J. L. Paniza Prados (eds), El Turismo en el Mediterráneo. Posibilidades de desarrollo y cohesión, Editorial Universitaria Ramón Areces, Madrid, 2009.

Savoja, L. (2010): La visita d'impresa come prodotto turistico. Una ricerca europea sui fattori di attrazione e le prospettive del turismo d'impresa, CCIIAA, Torino.

Urry, J. (1990): The Tourist Gaze, Sage, London.

Van den Berg, L. et al. (2010): Visita de empresa: una oportunidad para la ciudad y la empresa, in A. Zárate Martín \& J-R. Morice (eds.), Visita de empresa y turismo: contexto español y perspectivas europeas, Cámara de Comercio e Industria de Toledo, Toledo.

Zárate Martín, A. (2010): La visita de empresa en España: una modalidad en expansión, in A. Zárate Martín \& J-R. Morice (eds.), Visita de empresa y turismo: contexto español y perspectivas europeas, Cámara de Comercio e Industria de Toledo, Toledo. 